\title{
POTENCIAL REPRODUTIVO HORÁRIO DO PREDADOR DE LAGARTAS DESFOLHADORAS DO EUCALIPTO: Podisus nigrispinus (HETEROPTERA: PENTATOMIDAE) ${ }^{1}$
}

\author{
Evaldo Martins Pires², Rosenilson Pinto ${ }^{2}$, Mabio Chrisley Lacerda ${ }^{3}$, José Cola Zanuncio ${ }^{4}$ e Maria do \\ Carmo Queiroz Fialho ${ }^{2}$
}

\begin{abstract}
RESUMO - Insetos da subordem Heteroptera apresentam ampla diversidade faunística, incluindo predadores de pragas agrícolas e florestais. Espécies do gênero Podisus destacam-se entre os percevejos predadores no controle biológico de lagartas desfolhadoras de eucalipto, soja, algodão e tomate. O objetivo foi estudar o comportamento reprodutivo e a atividade de predação de Podisus nigrispinus (Heteroptera: Pentatomidae) alimentado com pupas de Tenebrio molitor L. (Coleoptera: Tenebrionidae) em laboratório. Casais desse predador foram acondicionados em potes plásticos de $500 \mathrm{~mL}$ com pupas de T. molitor e água em tubos tipo anestésico odontológico, inseridos na tampa desses potes. Foram observados: a postura o acasalamento e a alimentação de machos e fêmeas de $P$. nigrispinus às $0 \mathrm{~h}, 6 \mathrm{~h}, 12 \mathrm{~h}$ e $18 \mathrm{~h}$. O porcentual de fêmeas de $P$. nigrispinus predando foi de 26,$26 ; 24,39 ; 15,91$; e $34,21 \%$ e o de machos, de 7,$41 ; 6,20 ; 4,88$; e $5,77 \%$, às $0 \mathrm{~h}, 6 \mathrm{~h}$, $12 \mathrm{~h} \mathrm{e} 18 \mathrm{~h}$, respectivamente. O maior número de fêmeas ovipositando foi observado às $00 \mathrm{~h}$. A maior porcentagem de fêmeas predando foi às $18 \mathrm{~h}(32,21 \%)$ e a de atividade de postura, à $0 \mathrm{~h}$ (50,56\% dos ovos depositados), enquanto foram registrados apenas $1,66 \%$ dos ovos às $12 \mathrm{~h}$. O número de acasalamentos de P. nigrispinus foi maior às $12 \mathrm{~h}(34,58 \%)$, seguido das $00 \mathrm{~h}(29,65 \%), 18 \mathrm{~h}(22,36 \%)$ e $6 \mathrm{~h}(16,60 \%)$.
\end{abstract}

Palavras-chave: Controle biológico, ritmo horário, predador e manejo da criação massal.

\section{HOURLY REPRODUCTIVE POTENTIAL OF THE PREDATOR OF LEPIDOPTERA EUCALYPT DESFOLIATORS - Podisus nigrispinus (HETEROPTERA: PENTATOMIDAE)}

\begin{abstract}
Insects of the sub-order Heteroptera present a wide diversity including predators of agricultural and forest pests. Species of the genus Podisus are important agents of biological control of defoliating caterpillars of eucalyptus, soybem, cotton and tomato. The objective was to study the reproductive behavior and predation rate of Podisus nigrispinus (Dallas) (Heteroptera: Pentatomidae) fed with Tenebrio molitor L. (Coleoptera: Tenebrionidae) pupae in the laboratory. Pairs of this predator were placed in $500 \mathrm{ml}$ plastic pots with $\mathbf{T}$. molitor pupae and water in tubes of anesthetic odontologic type inserted in the lid. The egg masses were removed and matting and feeding of males and females $\boldsymbol{P}$. nigrispinus were observed at 12:00 AM, 06:00 AM, 12:00 PM and 06:00 PM. The percentages of females of P. nigrispinus predating were 26.26, 24.39, 15.91 and $34.21 \%$, and males 7.41, 6.20, 4.88 e 5.77\% at 12:00 AM, 6:O0AM, 12:00 PM and 06:00 PM, respectively. The largest number of females of this predator laying eggs was observed at 12:00 AM. The largest percentage of females predating was observed at 6:00 PM (32.21\%) and the maximum number of eggs masses laid at 12:00 AM (50.56\% of the eggs deposited) while only 1.66\% of them were registered at 12:00 AM. The number of mating of P. nigrispinus was higher at 12:00 PM (34.58\%) followed by those at 12:00 AM (29.65\%) 06:00 PM (22.36\%) and 06:00 AM (16.60\%).
\end{abstract}

Keywords: Biological control, activity schedule, predator and mass rearing.

\footnotetext{
${ }^{1}$ Recebido em 22.02.06 e aceito para publicação em 23.08.2006.

2 Programa de Pós-Graduação em Entomologia da Universidade Federal de Viçosa. E-mail: <evaldo.pires@ gmail.com>.

${ }^{3}$ Programa de Pós-Graduação em Fitotecnia da Universidade Federal de Viçosa.

${ }^{4}$ Departamento de Biologia Animal/Entomologia da Universidade Federal de Viçosa. E-mail: <zanuncio@ufv.br>.
} 


\section{INTRODUÇÃO}

O controle biológico é importante para reduzir a dependência do uso de produtos químicos e restabelecer o equilíbrio entre insetos-praga e seus inimigos naturais (METCALF e LUCKMANN, 1982).

Insetos da subordem Heteroptera se destacam pela diversidade faunística (PAULA e FERREIRA, 1998) com espécies predadoras de pragas agrícolas e florestais (ZANUNCIO et al., 1994), os quais representam a primeira linha de defesa de plantas contra fitófagos (WHITCOMB, 1981).

Os predadores Pentatomidae têm alta fecundidade (MEDEIROS et al., 2000) em plantios florestais onde insetos fitófagos podem causar danos (SANTOS et al., 1996; ZANUNCIO et al., 1994) pela grande disponibilidade de alimento e baixas populações de inimigos naturais (ASSIS Jr. et al., 1998). Isso torna necessário o aprimoramento de técnicas de criação massal de predadores para liberação em áreas com surtos populacionais de insetos-praga (LACERDA et al., 2004).

Espécies do gênero Podisus apresentam ampla distribuição (De CLERCQ, 2000; THOMAS, 1992) e têm sido usadas para o controle biológico de lagartas desfolhadoras de eucalipto (ZANUNCIO et al., 1993), algodão (MEDEIROS et al., 2000), soja (MATOS NETO et al., 2002) e tomate (Oliveira et al., 2002), o que torna necessário estudar a biologia, a ecologia, a sistemática e as técnicas de criação desses inimigos naturais (GRAZIA e HILDEBRAND, 1987).

O período de postura de $P$. nigrispinus foi dividido em picos de oviposição, quando $50 \%$ das fêmeas já fizeram a primeira postura e finalizaram quando $60 \%$ dos ovos foram colocados (MEDEIROS et al., 2000, 2003). Isso ocorre, aproximadamente, entre o 20 e $30^{\circ}$ dias da fase adulta desse predador.

O objetivo foi estudar o comportamento reprodutivo e a atividade de predação de $P$. nigrispinus (Heteroptera: Pentatomidae) alimentado com pupas de Tenebrio molitor L. (Coleoptera: Tenebrionidae) em laboratório.

\section{MATERIAL E MÉTODOS}

A pesquisa foi desenvolvida no Laboratório de Controle Biológico do Instituto de Biotecnologia Aplicada à Agropecuária (BIOAGRO) da Universidade Federal de Viçosa (UFV), em Viçosa, Estado de Minas Gerais, à temperatura de $25 \pm 2{ }^{\circ} \mathrm{C}$ e umidade relativa de 70 $\pm 10 \%$ e fotoperíodo de $12 \mathrm{~h}$.

R. Árvore, Viçosa-MG, v.30, n.6, p.1039-1044, 2006
Ninfas de P. nigrispinus foram obtidas da criação massal desenvolvida no Insetário do Departamento de Biologia Animal da UFV. Foram utilizadas 20 repetições, sendo cada unidade amostral representada por grupo de 10 indivíduos por placa de Petri (diâmetro $=15 \mathrm{~cm}$, altura $=1,2 \mathrm{~cm}$ ) e durante a fase ninfal. Essas ninfas receberam, a partir do segundo estádio, pupas de $T$. molitor e água em tubos tipo anestésico odontológico de $2,5 \mathrm{ml}$.

Os adultos de $P$. nigrispinus foram sexados, pela aparência externa da genitália, logo após a emergência e selecionados os casais. As fêmeas desse predador foram separadas por três dias em copos plásticos de $500 \mathrm{ml}$ (ZANUNCIO et al., 1992) e, posteriormente, colocadas em duas gaiolas de $30 \times 30 \mathrm{~cm}$ com os machos, para se acasalarem. Os casais em cópula foram retirados dessas gaiolas e acondicionados em copos plásticos de $500 \mathrm{ml}$, sendo utilizados 50 casais, individualizados em copos. Duas pupas de T. molitor foram substituídas, a cada 6 h, em cada copo, além de água.

As observações foram iniciadas 30 dias após a emergência dos adultos de P. nigrispinus, quando a oviposição desse predador atinge o máximo (MEDEIROS et al., 2000, 2003) com duração de 10 dias consecutivos, quando foram observados o acasalamento, as posturas, e a alimentação de machos e fêmeas desse predador, às $00 \mathrm{~h}, 06 \mathrm{~h}, 12 \mathrm{~h}$ e $18 \mathrm{~h}$.

Os dados de postura, acasalamento e alimentação de machos e fêmeas de $P$. nigrispinus foram submetidos à análise de variância e as médias, comparadas pelo teste de Tukey a $5 \%$ de probabilidade.

\section{RESULTADOS E DISCUSSÃO}

P. nigrispinus teve maior oviposição, no intervalo das 18 às $24 \mathrm{~h}, \operatorname{com} 50,56 \%$ dos ovos colocados, enquanto isso foi de apenas $1,66 \%$ entre 06 e $12 \mathrm{~h}$ (Figura 1), o que discorda do fato de que insetos do gênero Podisus são de hábitos diurnos (HUTCHINS et al., 2003). No entanto, isso varia com a espécie ou grupo de insetos, pois Phyllocnistis citrella Stainton (Lepidoptera: Gracillariidae) teve $98,9 \%$ dos ovos colocados das 20 $\mathrm{h}$ às $08 \mathrm{~h}$, quando a falta de luminosidade aumentou a reprodução desse lepidóptero (PARRA et al., 2002). Isso é comum para lepidópteros com hábito noturno, cuja reprodução aumenta ao entardecer e durante a noite (MEYER, 1969). 
Os horários de oviposição, acasalamento e alimentação de predadores devem ser conhecidos, pois o manuseio nesses períodos pode prejudicar seu desempenho e reduzir sua capacidade reprodutiva. Além disso, a luz pode afetar a reprodução dos insetos com parte deles se reproduzindo no escuro e outros sob luz direta (GALLO et al., 2002). De forma semelhante, o acasalamento pode ocorrer no claro ou escuro, dependendo da espécie. P. nigrispinus apresentou comportamento reprodutivo pouco definido (CARVALHO et al., 1994), o que concorda com relatos de ritmo diurno e melhor horário de acasalamento às 12 h (34,58\%). Isso coincidiu com o menor número de machos e fêmeas predando e de postura e oviposição, nesse horário. A freqüência de acasalamento de $P$. nigrispinus foi menor às $6 \mathrm{~h}(16,60 \%)$, seguido pelos de 18 e $00 \mathrm{~h}$, com 22,36 e $29,65 \%$ dos casais acasalando, respectivamente (Figuras 1, 2 e 3 ).

O menor número de acasalamentos de $P$. nigrispinus (Figura 3) concorda com o relatado para esse predador ( Podisus connexivus $=P$. nigrispinus $)$, das 9 às 11 h 59 (CARVALHO et al., 1994). Isso mostra que o período da manhã é mais favorável para o manejo da criação massal de $P$. nigrispinus em laboratório, quando esse predador apresenta menores valores de acasalamento e postura. No entanto, a freqüência de acasalamento de P. nigrispinus difere do relatado para Brontocoris tabidus (Signoret) e P. nigrispinus (Heteroptera: Pentatomidae), sem preferência para intervalos horários (FERNANDES et al., 1996), o que pode ser devida à não-avaliação num período de $6 \mathrm{~h}$.

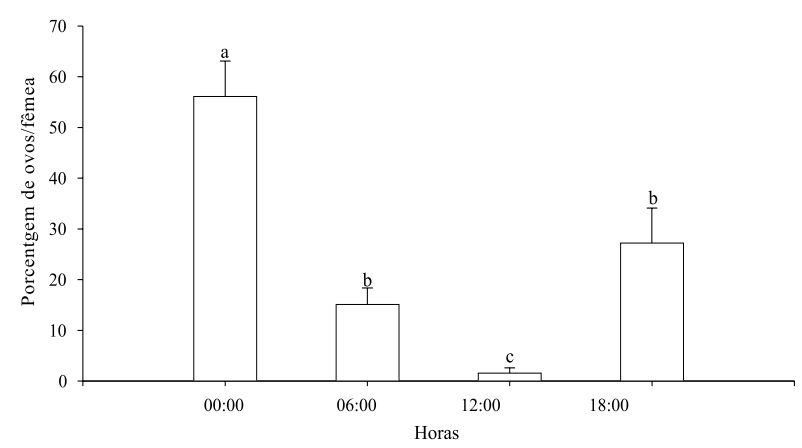

Figura 1 - Porcentagem de ovos por fêmea de Podisus nigrispinus (Heteroptera: Pentatomidae) em diferentes períodos diários. $\left(25 \pm 2{ }^{\circ} \mathrm{C}, 70 \%\right.$ UR e fotofase de $12 \mathrm{~h}$ ).

Figure 1 -Percentage of eggs per female of Podisus nigrispinus (Heteroptera: Pentatomidae) at different hours $\left(25 \pm 2{ }^{\circ} \mathrm{C}, 70 \% \mathrm{RH}\right.$ and photo phase of 12 hours $)$.

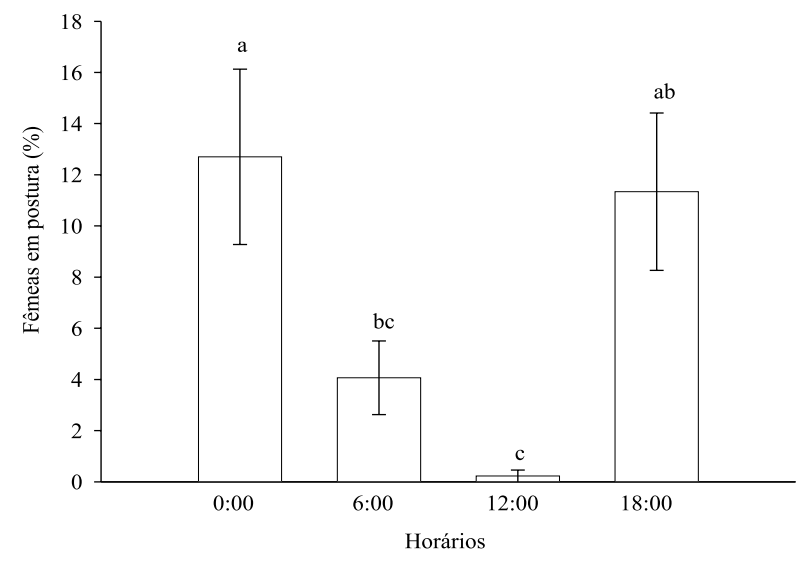

Figura 2 - Porcentagem de Podisus nigrispinus (Heteroptera: Pentatomidae) ovipositando às $0 \mathrm{~h}, 6 \mathrm{~h}, 12 \mathrm{~h}$ e $18 \mathrm{~h}$, em laboratório $\left(25 \pm 2{ }^{\circ} \mathrm{C}, 70 \%\right.$ UR e fotofase de $12 \mathrm{~h})$.

Figure 2 - Percentage of Podisus nigrispinus (Heteroptera: Pentatomidae) females laying eggs at 12:00 AM, 06:00 AM, 12:00 PM and 06:00 PM in the laboratory $\left(25 \pm 2^{\circ} \mathrm{C}, 70 \% \mathrm{RH}\right.$ and photo phase of 12 hours $)$.

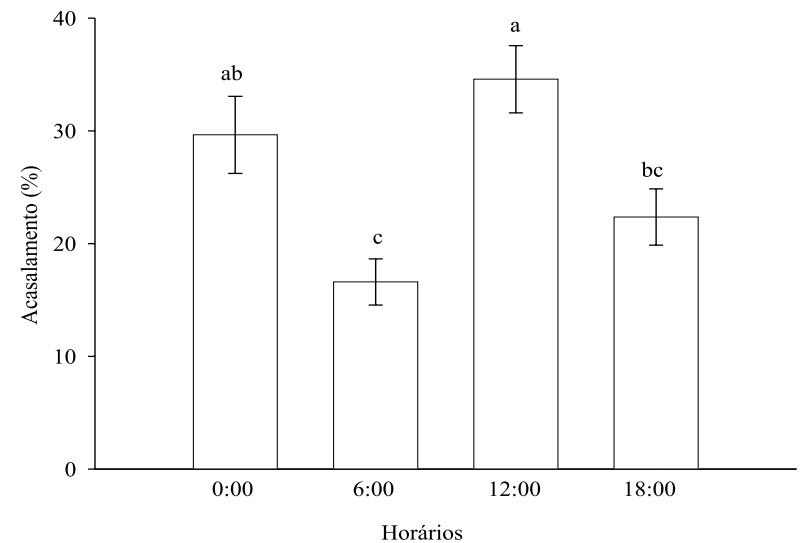

Figura 3 - Porcentagem de fêmeas de Podisus nigrispinus (Heteroptera: Pentatomidae) acasalando às $0 \mathrm{~h}$, $6 \mathrm{~h}, 12 \mathrm{~h}$ e $18 \mathrm{~h}$, em laboratório, $\left(25 \pm 2{ }^{\circ} \mathrm{C}\right.$, $70 \%$ UR e fotofase de 12 horas).

Figure 3 - Percentage of females of Podisus nigrispinus (Heteroptera: Pentatomidae) mating at 12:00 AM, 06:00 AM, 12:00 PM and 06:00 PM in the laboratory $\left(25 \pm 2{ }^{\circ} \mathrm{C}, 70 \% \mathrm{RH}\right.$ and photo phase of 12 hours $)$.

O período de incubação dos ovos de $P$. nigrispinus foi de 5,0 $\pm 0,25$ dias, com taxa de viabilidade dos ovos de $87,94 \%$, sendo semelhante ao relatado para esse predador, com 79,25 e 85,19\% de eclosão dos ovos quando alimentado com Tenebrio molitor (Coleoptera: Tenebrionidae) e Spodoptera frugiperda (Lepidoptera: Noctuidae), respectivamente (OLIVEIRA et al., 2004)

R. Árvore, Viçosa-MG, v.30, n.6, p.1039-1044, 2006 
A retirada dos ovos a cada seis horas pode ter reduzido a predação destes, pois esta pode ser mais intensa durante períodos com falta de alimento (MOLINARUGAMA et al., 1998). Dessa forma, isso não foi pronunciado neste trabalho, pois os insetos eram alimentados e os ovos retirados diariamente.

Os porcentuais de fêmeas de $P$. nigrispinus predando foram de 26,26; 24,39; 15,91; e 34,21\%, às 00, 06, 12 e $18 \mathrm{~h}$, respectivamente, mas os machos apresentaram menor porcentual de indivíduos predando que as fêmeas, com 7,$41 ; 6,20 ; 4,88$; e 5,77\% nesses horários. Esse menor porcentual no caso dos machos pode se devido ao fato de as fêmeas necessitarem de maior quantidade de energia para a postura (ZANUNCIO et al., 2002).

O melhor horário para se manejar a criação de $P$. nigrispinus é a parte da manhã para minimizar o impacto sobre as fêmeas em postura, acasalamento e, ou, predação e, conseqüentemente, o estresse sobre esse predador.

No campo, tais informações sobre os períodos de atividade mais intensas desse predador podem auxiliar a metodologia de manejo nessas condições, por exemplo aplicação a de produtos visando controlar determinada praga da cultura. Nessa situação hipotética, deve-se preservar os horários de maior atividade do predador.

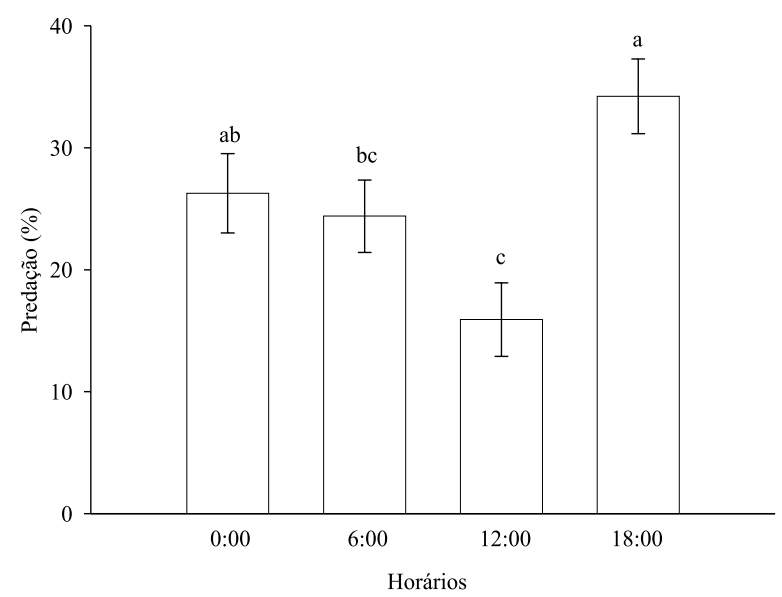

Figura 4 - Porcentagem de fêmeas de Podisus nigrispinus (Heteroptera: Pentatomidae) predando pupas de Tenebrio molitor (Coleoptera: Tenebrionidae) às $0 \mathrm{~h}, 6 \mathrm{~h}, 12 \mathrm{~h}$ e $18 \mathrm{~h}$, em laboratório $(25 \pm 2$ ${ }^{\circ} \mathrm{C}, 70 \%$ UR e fotofase de 12 horas).

Figure 4-Percentage offemales of Podisus nigrispinus (Heteroptera: Pentatomidae) preying Tenebrio molitor (Coleoptera: Tenebrionidae) pupae at 12:00 AM, 06:00 AM, 12:00 $P M$ and $06: 00 P M$ in the laboratory $\left(25 \pm 2{ }^{\circ} \mathrm{C}\right.$, $70 \%$ RH and photo phase of 12 hours).

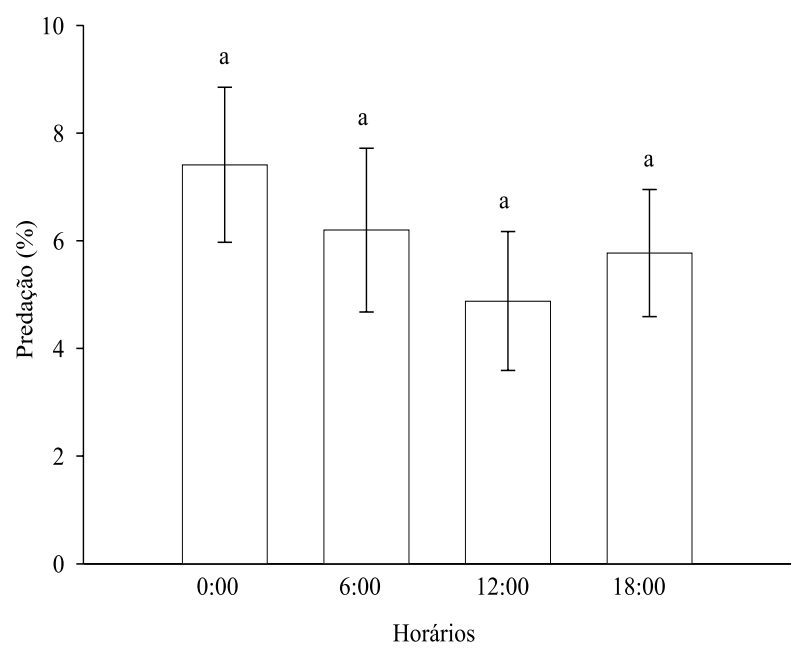

Figura 5 - Porcentagem de machos de Podisus nigrispinus (Heteroptera: Pentatomidae) predando pupas de Tenebrio molitor (Coleoptera: Tenebrionidae) às $0 \mathrm{~h}, 6 \mathrm{~h}, 12 \mathrm{~h}$ e $18 \mathrm{~h}$, em laboratório $(25 \pm 2$ ${ }^{\circ} \mathrm{C}, 70 \%$ UR e fotofase de 12 horas).

Figure 5 -Percentage of males of Podisus nigrispinus (Heteroptera: Pentatomidae) predating Tenebrio molitor (Coleoptera: Tenebrionidae) pupae at 12:00 AM, 06:00 AM, 12:00 PM and 06:00 PM in the laboratory $\left(25 \pm 2{ }^{\circ} \mathrm{C}, 70 \% \mathrm{RH}\right.$ and photo phase of 12 hours).

\section{AGRADECIMENTOS}

A Coordenação de Aperfeiçoamento de Pessoal de Nível Superior (CAPES), ao Conselho Nacional de Desenvolvimento Científico e Tecnológico (CNPq) e a Fundação de Amparo à Pesquisa do Estado de Minas Gerais (FAPEMIG).

\section{REFERÊNCIAS BIBLIOGRÁFICAS}

ASSIS JR, S.L. et al. Efeito da suplementação de folhas de Eucalyptus urophylla no desenvolvimento e reprodução do predador Supputius cincticeps (Stal) (Heteroptera: Pentatomidae). Anais Sociedade Entomológica do Brasil, v. 27, n. 2, p. 245-253, 1998.

CARVALHO, R.S. et al. Ritmo do comportamento de acasalamento e atividade sexual de Podisus connexivus Bergroth (Heteroptera: Pentatomidae: Asopinae). Anais Sociedade Entomológica do Brasil, v. 23, n. 2, p. 197-202, 1994. 
DE CLERCQ P. Predaceous stinkbugs (Pentatomidae: Asopinae). In: SCHAEFER, C.W; PNIZZI, A.R. (Eds.). Heteroptera of economic importance. Cambridge: Cambridge University Press, 2000. p. 737-789.

FERNANDES, L.G. et al. Aspectos biológicos de Brontocoris tabidus Signoret, 1852 e Podisus nigrispinus Dallas, 1851 (Heteroptera: Pentatomidae). Revista Cerne, v. 2, n. 1, p. 1-10, 1996.

GALLO, D. et al. Entomologia agrícola. Piracicaba: FEALQ, 2002.920 p.

GRAZIA, J.; HILDEBRAND, R. Hemípteros predadores de insetos. In: ENCONTRO SUL BRASILEIRO DE CONTROLE BIOLÓGICO DE PRAGAS, 1986, Passo Fundo. Anais... Passo Fundo: AEAPF-CNPQ/EMBRAPA, 1987. p. 21-37,

HUTCHINS, M. et al. (Eds.). Grzimek's Animal Life Encyclopedia, 2. ed. Insects. Farmington Hills, MI: Gale Group, 2003. v. 3, 489 p.

LACERDA, M.C. et al. Development and reproduction of Podisus distinctus (Heteroptera: Pentatomidae) fed larva of Bombyx mori (Lepidoptera: Bombycidae). Brazilian Journal of Biolology, v. 64, n. 2, p. 237-242, 2004.

MATOS NETO F.C. et al. Reproductive characteristics of the predator Podisus nigrispinus fed with an insect resistant soybean variety. Pesquisa Agropecuária Brasileira, v. 37, n. 7, p. 917-924, 2002.

MEDEIROS, R.S. et al. Age-dependent fecundity and life-fertility tables for Podisus nigrispinus (Dallas) (Het., Pentatomidae). Journal of Applied Entomology, v. 124, n. 7-8, p. 319-324, 2000.

MEDEIROS, R.S. et al. Effect of temperature on life table parameters of Podisus nigrispinus (Het., Pentatomidae) fed with Alabama argillacea (Lep., Noctuidae) larvae. Journal of Applied Entomology, v. 127, n. 4, p. 209-213, 2003.

METCALF, R.L.; LUCKMANN, W.H. Introduction to insect pest management. New York: John Wiley \& Sons, 1982.328 p.
MEYER, D. Der einfluss von licht und temperaturschwankungen auf verhalten und fekundität des lärchenwicklers Zeiraphera diniana (Gn.) (Lepidoptera: Tortricidae). Revue Suisse de Zoologie, v. 76, p. 93-141, 1969.

MOLINA-RUGAMA, A.J. et al. Efecto de la escasez de alimento en la reproducción y longevidad de Podisus rostralis (Stal) (Heteroptera: Pentatomidae: Asopinae). Tropical Ecology, v. 39, n. 2, p. 185-191, 1998.

OLIVEIRA, J.E.M. et al. Efeito das plantas do algodoeiro e do tomateiro, como complemento alimentar, no desenvolvimento e na reprodução do predador Podisus nigrispinus (Dallas) (Heteroptera: Pentatomidae).

Neotropical Entomology, v. 31, n. 1, p. 101-108, 2002.

OLIVEIRA, H.N. et al. Desenvolvimento do predador Podisus nigrispinus alimentado com Spodoptera frugiperda e Tenebrio molitor. Pesquisa Agropecuaria Brasileira, v. 39, n. 10, p. 947-951, 2004.

PARRA, A.L.G.C.; VILELA, E.F.; BENTO, J.M.S. Horário de oviposição e ritmo diário de emergência de Phyllocnistis citrella Stainton (Lepidoptera: Gracillariidae) em laboratório. Neotropical Entomology, v. 31, n. 3, p. 365-368, 2002.

PAULA, A.S.; FERREIRA, P.S.F. Fauna de Heteroptera de la Mata do Corrego do Paraiso, Vicosa, Minas Gerais, Brasil. I. riqueza y diversidad especificas. Anales del Instituto Biologico de la Universidad Nacional Autonoma del Mexico, Serie Zoologica, v. 69, p. 39-51, 1998.

SANTOS, G.P.; ZANUNCIO, J.C.; ZANUNCIO, T.V. Pragas do eucalipto. Informe Agropecuário, v.18, n. 185, p. 63-71, 1996.

THOMAS, D.B. Taxonomic synopsis of the Asopinae Pentatomidae (Heteroptera) of the Western Hemisphere. Lanham: Entomologial Society of America, 1992. 156 p.

R. Árvore, Viçosa-MG, v.30, n.6, p.1039-1044, 2006 
ZANUNCIO, J.C. et al. Determinação da idade ideal para o acasalamento de fêmeas de Podisus connexivus Bergroth, 1891 (Hemiptera:

Pentatomidae) visando uma criação massal.

Revista Árvore, v. 16, n. 3, p. 362-367, 1992.

ZANUNCIO, J.C. et al. Impact of two formulations of deltamethrin in aerial application against eucalyptus caterpillars and their predaceous bugs. Mededelingen

Faculteit Landbouwkundige en

Toegepaste Biologische

Wetenschappen Universiteit Gent, v. 58, n. 2, p. 477-481, 1993.
ZANUNCIO, J.C. et al. Hemipterous predators of eucalypt defoliator caterpillars. Forest Ecology Management, v. 65, n. 1, p. $65-73,1994$.

ZANUNCIO, J.C. et al. Effect of body weight on fecundity and longevity of the stinkbug Podisus rostralis. Pesquisa Agropecuária

Brasileira, v. 37, n. 9, p. 1125-1230, 2002.

WHITCOMB, W.H. The use of predators in insect control. In: PIMENTEL, D. (Ed.). CRC handbook of pest management in agriculture Boca Raton: CRC, 1981.p. 105-123. 